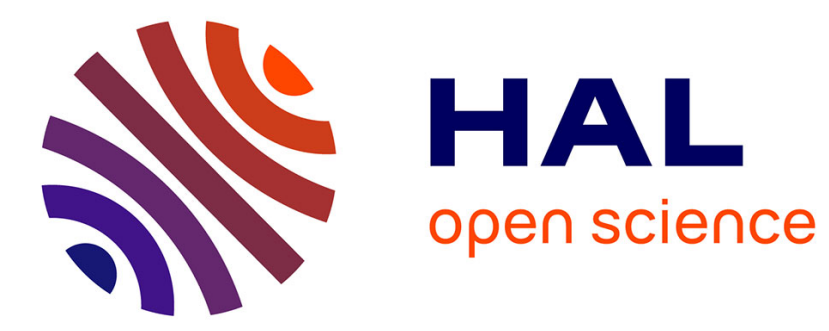

\title{
Damage at heterogeneous interfaces
}

\author{
Stéphane Roux, Arnaud Delaplace, Gilles Pijaudier-Cabot
}

\section{To cite this version:}

Stéphane Roux, Arnaud Delaplace, Gilles Pijaudier-Cabot. Damage at heterogeneous interfaces. Physica A: Statistical Mechanics and its Applications, 1999, 270 (1-2), pp.35-41. 10.1016/S03784371(99)00154-5 . hal-01006713

\section{HAL Id: hal-01006713 https://hal.science/hal-01006713}

Submitted on $30 \mathrm{Jul} 2017$

HAL is a multi-disciplinary open access archive for the deposit and dissemination of scientific research documents, whether they are published or not. The documents may come from teaching and research institutions in France or abroad, or from public or private research centers.
L'archive ouverte pluridisciplinaire HAL, est destinée au dépôt et à la diffusion de documents scientifiques de niveau recherche, publiés ou non, émanant des établissements d'enseignement et de recherche français ou étrangers, des laboratoires publics ou privés. 


\title{
Damage at heterogeneous interfaces
}

\author{
Stéphane Roux ${ }^{\mathrm{a}}$, Arnaud Delaplace ${ }^{\mathrm{b}}$, Gilles Pijaudier-Cabot ${ }^{\mathrm{b}}$ \\ ${ }^{a}$ Laboratoire Surface du Verre et Interfaces, Unité Mixte de Recherche CNRS Saint-Gobain, \\ 39 Quai Lucien Lefranc, 93303 Aubervilliers Cedex, France \\ bLaboratoire de Mécanique et Technologie, Ecole Normale Supérieure de Cachan \\ n61 Avenue du Président Wilson, 94235 Cachan Cedex, France
}

We discuss the progressive damage of an heterogeneous interface modelled through a fiber bundle with random strength, coupled to two semi-in finite elastic blocks. We show that a hierarchical description of the elastic blocks and an homogeneous damageable interface gives rise to a cascade of bifurcations associated to the progressive concentration of damage activity of a narrower and narrower domain starting from half the system size. The disorder in the limit of an infinite size of the elastic block is a weak relevance for the global effective interface law.

Keywords: Fracture; Damage; Localization; Fiber bundles; Size effects

\section{Introduction}

The success of continuum mechanics approaches to have access to an accurate description of the behavior of homogeneous and disorder media is impressive. However, the tools which have been developed so far are based on macroscopic experimental observations, and little progress has been achieved so far in justifying the foundations of the mechanical behavior from a local description which incorporates disorder. This is especially true for damage mechanics [1]. The latter describes a regime where numerous micro-cracks are created in the material under loading, and hence the elastic properties are progressively reduced. However, for these micro-cracks to be stable, i.e. that they do not grow in an unstable fashion under constant loading, heterogeneities have to be present. Without them, a brittle behavior would result. No heterogenity is explicitly included in damage mechanics, and thus one cannot distinguish a priori 
between a brittle or a damage behavior (see e.g. Ref. [2] for a specific discussion devoted to concrete).

The aim of our study is to shed some light on the transition from damage to brittle behavior, from a theoretical point of view. In order to do so, we will introduce a very simple model for a damageable interface coupled to an elastic block. Such a simple model allows us to treat explicitly the presence of heterogeneities, and thus to investigate their role in details. Other more elaborate models have been proposed in the literature [4], but they generally force one to consider numerical simulations from which extrapolations to large system sizes may be uncertain. Under some circumstances to be discussed below, an homogeneous loading of an homogeneous material described by a damage behavior can lead to a bifurcation point where different evolutions are possible each of them being a solution of the problem [3]. Among these solutions, some generally correspond to a localized mode for the strain increment (and thus damage increment) fields. Hence the term of "localization" used in this context. This localization point is thus not determined by heterogeneities although it may appear in practice as a transition from a uniform damage mode to a localized one where damage condenses on a surface, which thus shares a number of similarities with brittle fracture. Quite generally, this bifurcation point is also a point where the system loses its stability [3]. Hence in the vicinity of this point, the medium becomes highly sensitive to any perturbation, and therefore heterogeneities may again become relevant in the description.

This very general discussion raised the issue of the relevance of disorder in the macroscopic behavior, and thus also the key question of the physical length scales involved at various stages of the loading. In order to progress along these lines, we deliberately choose a simple case, which gives a concrete way to address these questions.

\section{Daniels' model}

Daniels' model [5] provides us with such a toy model, with both an extreme simplicity and still not trivial behaviors, in particular for extensions of the original model where elastic couplings are introduced. Let us consider a one dimensional array of elastic-brittle fibers, each having a uniform stiffness, $k$, but a random distribution of failure load $f_{c}$, chosen here as a uniform distribution between 0 and $F_{m}$. Similarly the stiffness can be set equal to unity without any loss of generality. A large number $N$ of such fibers are placed in parallel between two rigid plates, where either a force $F$ or a displacement $U$ is imposed. We define the stress as $\sigma \equiv F / N$. The mean behavior is simple to compute and was proposed by Daniels [5] as early as in the 1940s:

$$
\langle\sigma(U)\rangle=\frac{k U\left(F_{m}-k U\right)}{F_{m}} .
$$

The stress-displacement relation is therefore a simple parabola. Using different distributions of failure forces, any other damage law can be implemented. For any finite 
sample, the observed response will display fluctuations around this curve. These fluctuations are the signature of the heterogeneous nature of the fibers, and thus it calls for some comments.

The first observation is that it is simple to compute the probability that the stress deviates from the mean behavior. At a fixed $U, \sigma$ is Gaussian distributed (for large $N$ ). The variance $\delta^{2}$ of the distribution is

$$
\frac{\delta^{2}}{\langle\sigma(U)\rangle}=\frac{1}{2 N} \frac{k U\left(F_{m}-k U\right)}{F_{m}^{2}+k U\left(F_{m}-k U\right)}=\frac{1}{2 N} \frac{\langle\sigma(U)\rangle}{F_{m}+\langle\sigma(U)\rangle}
$$

and thus asymptotically, as $N$ goes to infinity, the fluctuations vanishes as $1 / \sqrt{N}$. We will see below that in spite of this vanishing amplitude, fluctuations can still play a role at the macroscopic level.

The amplitude of fluctuations from sample to sample does not exhaust the characterisation of these fluctuations. An essential feature is to characterise the fluctuations along the response. The question is thus the following: let us assume that we know that the fiber bundle has reached a particular state $(U, \sigma(U))$. What can be said about $\sigma(U+d U)$ ? Skipping over computational details, one can show that the problem becomes equivalent to a random walk problem with a local bias, $d\langle\sigma\rangle / d U$. Then, the response is not regular, as for a number of mechanical problems where disorder is taken into account.

Let us suppose that a constant force is imposed on the system. From the average law, we see that the fiber bundle will resist any stress less then $\sigma^{*}=k U / 2$, which is reached for a displacement $U^{*}=F_{m} /(2 k)$. In order to analyse the effective response of a finite size system, we need to take into account the fluctuations of the response. The above analysis revealed that the increments of the mechanical response is a biased random walk, and thus this analogy can be exploited here. At constant force, a number $\Delta$ of fibers can be broken simultaneously. This constitutes an avalanche. This number of broken fiber in an avalanche is given by the probability that the biased random walk returns to its initial value after $\Delta$ steps. For small steps, the bias can be ignored and thus $\Delta$ is distributed as a power-law $p(\Delta) \propto \Delta^{-3 / 2}$. However, after a distance such that the bias becomes of order of the fluctuation, $p(\Delta)$ vanishes exponentially fast. This characteristic size $\Delta^{*}$ is thus of order $(d \sigma / d U) \Delta^{*} / N \propto \sqrt{\Delta^{*}} / N$ or using $d \sigma / d U \propto\left(U^{*}-U\right)$

$$
\Delta^{*} \propto\left(U^{*}-U\right)^{-2} .
$$

Therefore the distribution of avalanches takes the form

$$
p(\Delta)=\Delta^{-3 / 2} \Phi\left(\frac{\Delta}{\left(U^{*}-U\right)^{-2}}\right),
$$

where $\Phi$ is a scaling function which is a constant for small arguments, and which vanishes exponentially for arguments larger than 1 . We have derived this result using only scaling arguments. A much more detailed analysis has been proposed a few years ago by Hemmer and Hansen [6,12] who derived the exact analytic expression for this distribution. It is important to underline that taking into account the disorder in 
the system has profoundly modified the picture of the approach to failure. Instead of the binary (resist/fail) response obtained from the average response, we have seen that the response of a finite size system displays a rich statistics of displacement increments which inform on the proximity of macroscopic failure. Furthermore, the regularity (or rather lack of regularity) forbids the use of standard criterion based on tangent stiffness.

The statistics of these avalanches describes the jumps in the displacement as the load is slightly increased. These jumps are counted in units of individual fibers, and thus the smaller jumps are expected to be hard to measure experimentally. Nevertheless, as one approaches the peak stress, the divergence of $\Delta^{*}$ is such that avalanches reach a macroscopic size. In order to illustrate the use of this result, let us study the fluctuation of the displacement $V=\left(U^{*}-U\right)$ at the maximum force. This displacement corresponds to an avalanche of size $\Delta=N V$. If such an avalanche occurs with a non-vanishing probability, it will allow for an increment of the displacement sufficient for leading to failure at a constant force. This provides the solution when using the expression of $\Delta^{*}: N V=V^{-2}$ or

$$
V \propto N^{-1 / 3} \text {. }
$$

This result which is non trivial cannot be obtained without taking into account the correlations of the noise about the mean response, in contrast to the fluctuation of the peak stress. This also shows that the largest avalanche to be observed is of order $N V$, increasing as $N^{2 / 3}$ with the system size.

\section{Coupling to an elastic block}

The weakness of Daniels' model comes from the infinitely rigid bar which couples all fibers. The opposite limit of extremely compliant bars has been proposed by Phoenix [7] and studied extensively in particular by Leath et al. [8]. In this limit, when a fiber breaks, the force it supported is transferred to the two closest surviving fibers (such a rule is called "Local Load Sharing" (LLS) as opposed to the "Global Load Sharing" (GLS) of Daniels' model). The behavior observed in this model is very different from the previous case. After a short initial phase, a critical defect (i.e. a few broken bonds) is nucleated. This defect grows and leads to a "brittle" failure. Let us mention that the statistics of avalanches has also been obtained [9] for this model, and not surprisingly, even if the statistics is recorded up to the failure point, the distribution is not algebraic for large avalanche sizes. Thus as neither of these two limits (infinitely stiff or compliant bar) is applicable, the question of the behavior of the system for intermediate stiffness is of crucial interest.

The fibers are imagined to be connected to two semi-infinite elastic blocks instead of the rigid bars of the original Daniels' model. This introduces a non-local coupling between the fibers: if one fiber breaks, the load has to be distributed over the other fibers balancing the elastic stress in the blocks and the tension in the fibers. We use a hierarchical decomposition of the blocks, which allows to solve for the stress distribution 
in a very simple way, and however to preserve the essential features of the continuum block description (such as the scaling of the Green function with the distance to the point source). We refer the reader to Ref. [10] for a more detailed discussion of the results and their derivations. Using the average interface behavior given through Eq. (1), we found that for a large size of the elastic blocks, a cascade of bifurcations occurred leading to a progressive concentration of the damage activity on a smaller and smaller part of the interface. The first bifurcation takes place at a point which approaches the apex of the stress/displacement curve. Past this point, damage continues to progress on one half of the interface, while the other half is elastically unloaded. Thus the first instability has the largest wavelength admissible in the system. As the displacement at infinity is increased, the interface encounters a second bifurcation where damage concentrates on a quarter of the interface, and the other parts are elastically unloaded. A third instability occurs after some increment of the displacement at infinity, and so on down to the stage where damage activity is confined to the smallest scale available. All the bifurcation points can be analytically computed. One can define an Equivalent Homogeneous Interface (EHI) response such that the global behavior of the elastic blocks and the interface is the same. Prior to the first bifurcation, the EHI behavior is identical to the real interface. However, as the damage becomes inhomogeneous, this is no longer true. This EHI response can again be solved analytically.

At the "final" stage of the bifurcation cascade, the damage along the interface reveals the evolution of the damage localization: as one moves away from the final active zone, the damage decreases as the inverse of the distance down to the value at the onset of the first bifurcation. This final stage corresponds to a complete damage (all fibers broken) in one element. After this, a crack is initiated and propagates through this inhomogeneous damage landscape. The crucial feature of this localization scenario is that failure is controlled by the largest scales. However, we have not yet included the presence of disorder along the interface. Thus the question which remains to be answered is whether the heterogeneous interface will follow a similar evolution or if nucleation of a critical defect may occur as in the LLS model. Indeed, the macroscopic response cannot answer this question directly.

We propose a simple argument to answer this question. Let us consider a displacement field along the interface which is harmonic of amplitude $A$ and wavelength $\lambda$. In terms of scaling, (i.e. ignoring dimensionless prefactor) the elastic energy of the elastic block per unit length of the interface (and for a thickness $z$ ) is $\mathscr{E}_{1}=E A^{2} z / \lambda$ (where $E$ is the Young modulus of the elastic blocks) as compared to the elastic energy of the interface $\mathscr{E}_{2}=n k A^{2}$ where $n$ is the number of fiber per unit length. Thus we see that the "stiffness" of the elastic block decreases for long wavelength modes. Therefore, it can be understood easily that short wavelength instabilities similar to the LLS limit may be prevented by a rather stiff interface in the short wavelength range. On the other hand, the nucleation of a critical defect could be considered over a larger scale. However, in this case, close to a defect, the redistribution of the load is shared over a length scale $\xi \propto E z /(k n)$. Then effective properties of segments of the interface of length $\xi$ have to be taken into account, and the latter display weaker and weaker 
fluctuations as their size increases. However, if the elastic modulus of the elastic block is small, we may still observe the nucleation and unstable growth of a local defect. Setting the wavelength to the distance between fibers $\lambda=1 / n$, this requires $E z \ll k$. The two-dimensional description of the elastic block requires moreover that the thickness $z$ is at most of order $1 / n$, so that the condition for a possible nucleation is $E \ll k n$. To be more precise one should be able to write a criterion for the size of the critical defect, taking into account the variability of the local fiber strength. This information is however still lacking. In the opposite case, disorder is expected to play a weak role and thus the disorderless case treated above is expected to be representative of the behavior. Numerical simulations were performed [10] taking into account the random distribution of fiber strengths and indeed an excellent agreement was observed with the disorderless interface.

\section{Conclusions}

Let us here simply emphasise the main points mentioned up to now.

- The stress/displacement response of the Daniels' model (and other variants) is a non-differentiable function which can be decomposed in an average (size independent) response, and a fluctuating part which can be mapped on a random walk problem.

- The statistics of avalanches is essentially controlled from the lack of regularity of this fluctuating part. This statistics shows progressive development of larger and larger avalanches up to the point of instability.

- The statistics of avalanches allows to derive the scaling of size effects on the displacement at peak stress. The latter is an original result.

- Introducing an elastic coupling between fibers gives rise to a different behavior. In the absence of heterogeneity, a bifurcation cascade occurs following the first localization threshold. In this cascade, damage progressively condenses from half the interface to a point-like domain. Note that we have also performed [11] a direct analysis using a boundary integral formulation: the only difference with the hierarchical decomposition is that the progressive damage condensation occurs continuously. The cascade of bifurcations is thus an artefact of the discretization which however does not play any significant role.

- When disorder is taken into account, the interface follows the same evolution as obtained without fluctuations provided the elastic modulus of the elastic blocks is sufficiently large as compared to the stiffness of the fibers. In this case, the statistics of avalanches is similar to the one obtained in Daniels' model, with a diverging size occurring exactly at the onset of the first bifurcation.

- For sufficiently compliant elastic blocks, nucleation of a critical defect can however still be obtained, as in the local load sharing model. This point however deserves a further study. 


\section{Acknowledgements}

The present text benefited from enlightening discussions with Jean-Pierre Vilotte and Jean-Paul Ampuero, particularly concerning the question of nucleation of a critical defect in the interface model.

\section{References}

[1] J. Lemaitre, A Course on Damage Mechanics, Springer, Berlin, 1992.

[2] Z.P. Bažant, J. Planas, Fracture and Size effect in Concrete and Other Quasi-brittle Materials, CRC Press, Boca Raton, 1998.

[3] Z.P. Bažant, L. Cedolin, Stability of Structures, Oxford University Press, Oxford, 1991.

[4] H.J. Herrmann, S. Roux (Eds.), Statistical Models for the Fracture of Disordered Media, Elsevier, Amsterdam, 1990.

[5] H.E. Daniels, Proc. Roy. Soc. London A 183 (1945) 405-435.

[6] P.C. Hemmer, A. Hansen, J. Appl. Mech. 59 (1992) 909-914.

[7] D.G. Harlow, S.L. Phoenix, Int. J. Fract. 17 (1981) 601.

[8] B. Wu, P.L. Leath, Preprint (Condmat/9811044), 1998.

[9] M. Kloster, A. Hansen, P.C. Hemmer, Phys. Rev. E 56 (1997) 2615.

[10] A. Delaplace, S. Roux, G. Pijaudier-Cabot, Int. J. Solids Struct. 36 (1999) 1403-1426.

[11] A. Delaplace, S. Roux, G. Pijaudier-Cabot, Int. J. Fract. (1999), in press.

[12] A. Hansen, P.C. Hemmer, Phys. Lett. A 184 (1994) 394-396. 